\title{
EI Giro a la Izquierda en el Uruguay de los Años 2000
}

\section{Uruguay's Left Turn in the 2000s}

\author{
Fernanda Cimini \\ Universidade Federal de Minas Gerais (UFMG) \\ E-mail: fcimini@cedeplar.ufmg.br \\ Izabella Leal Miranda Aguiar \\ Universidade Federal de Minas Gerais (UFMG) \\ E-mail: leal-bella@ufmg.br
}

Resumen: El artículo indaga en qué medida el giro a la izquierda en Uruguay, en los 2000, puede ser considerado una coyuntura crítica. Para la fundamentación empírica, se utilizó el método de process-tracing, identificando las condiciones permisivas, productivas y reproductivas relacionadas. Se concluyó que las políticas de bienestar social, realizadas por Frente Amplio, estarán sujetas a restricciones económicas a largo plazo si no se realiza un proceso de redistribución de los recursos nacionales. Esto se debe a la asociación económicamente concesiva del partido al capital internacional, profundizando el proceso de primarización de la economía uruguaya y generando restricción para políticas universalistas.

Palabras Clave: Economía Política; Uruguay; Reformas Liberales; Giro a la Izquierda.

\begin{abstract}
The article explores the extent to which the left turn in Uruguay, in the 2000s, can be considered a critical juncture. For the empirical foundation, the process-tracing method was used, identifying the permissive, productive and reproductive conditions related. It was concluded that the social welfare policies, carried out by Frente Amplio, will be subject to long-term economic restrictions if a process of redistribution of national resources is not carried out. This is due to the economically concessional association of the party to international capital, deepening the process of primarization of the Uruguayan economy and generating restrictions for universalist policies..
\end{abstract}

Keywords: Political Economy; Uruguay; Liberal Reforms; Left Turn. 
CIMINI, Fernanda; AGUIAR, Izabella Leal Miranda. El Giro a la Izquierda en el Uruguay de los Años 2000.

\section{Introducción}

El artículo se apoya en el institucionalismo histórico para indagar en qué medida el giro a la izquierda en Uruguay, en los 2000, puede ser considerado una coyuntura crítica en el sentido de desencadenar nueva trayectoria en el enfrentamiento al status quo de las élites económicas. Tras un escenario de crisis e inestabilidades económicas, el partido Frente Amplio logró canalizar las insatisfacciones de las masas y de parte de las élites económicas, que se encontraban descontentas con las reformas. Al mismo tiempo, el partido buscaba construir un modelo de política socialmente preocupado, que se diferenciaba del modelo neoliberal por buscar una redistribución más equitativa de la riqueza nacional. El agravamiento de la competencia política, en torno a una plataforma más equitativa, parecía indicar el inicio de una nueva trayectoria en el combate a las desigualdades económicas en el país.

En concreto, el artículo investiga las condiciones permisivas, productivas y reproductivas asociadas al ascenso de la izquierda uruguaya, buscando identificar los componentes que componen ese momento crítico en la historia de Uruguay. Se prestará mayor atención a las condiciones precedentes, teniendo en cuenta la premisa adoptada por el trabajo de que el legado neoliberal influye directamente en la formación de las coaliciones de apoyo que crearán oportunidades y límites para la gobernanza a la izquierda.

Además de esta introducción, el artículo está dividido en cinco secciones y encuentra su desenlace en las consideraciones finales. En la primera sección se presenta el debate teórico sobre el giro a la izquierda en América Latina y su posible encuadramiento como coyuntura crítica. En la segunda sección se introduce brevemente el contexto anterior al giro a la izquierda en Uruguay. En la tercera sección se arroja luz sobre las condiciones permisivas al giro a la izquierda en Uruguay, destacándose las implicaciones de la situación económica del país, así como del proceso de desindustrialización, de desmantelamiento del Estado de bienestar social y de la configuración de sus elites, que confluyeron para esa coyuntura crítica. En la cuarta sección se abordan las condiciones productivas del giro a la izquierda, con foco en la capacidad de la agenda frenteamplista de canalizar las insatisfacciones nacionales a su favor. En la quinta sección se trata de los mecanismos de reproducción del giro a la izquierda, enfatizando fenómenos de orden económico y demográfico actuantes, concomitantemente, en los ámbitos

Revista Brasileira de Políticas Públicas e Internacionais, v.4, n.1, julho/2019, pp. 58-81. 
CIMINI, Fernanda; AGUIAR, Izabella Leal Miranda. El Giro a la Izquierda en el Uruguay de los Años 2000.

externo e interno, además de la contribución de la postura resiliente y progresista del partido Frente Amplio para la conformación de una nueva política económica y social. Por fin, el artículo concluye que, aunque haya engendrado importantes avances en lo que concierne a la edificación de un sólido Estado de bienestar social, la izquierda uruguaya estará sujeta a restricciones de orden económico por no haber sido capaz de estancar el proceso de primarización de la economía. De esta forma, en ausencia de un profundo proceso de redistribución de recursos nacionales, estarán amenazados los legados universalistas del gobierno.

\section{El Giro a la Izquierda como Coyuntura Crítica}

El ascenso de gobiernos de izquierda al poder en ocho países latinoamericanos, en menos de una década - Venezuela (1998); Chile (2000); Brasil (2002); Argentina (2003); Uruguay (2005); Bolivia (2005); Nicaragua (2006); Ecuador (2007) - reavivó el debate el enfrentamiento a la desigualdad económica en la región, ya que gran parte de la literatura destaca que la característica común de los gobiernos de izquierda latinoamericanos ha sido justamente la preocupación por la reducción de desigualdades económicas (Levitsky y Roberts 2011, Flores-Macias 2012, Huber y Stephens 2012, Weyland, Madrid y Hunter 2016). Entre las medidas adoptadas por los gobiernos de izquierda, con impacto en la reducción de las desigualdades, los tipos más comunes fueron (i) aumento en los gastos sociales, por medio de nuevos programas sociales (Bolsa Familia, Misiones); (ii) aumento de la cobertura de programas ya existentes y (iii) políticas con impacto en el mercado de trabajo (aumento real del salario mínimo y cambio en las condiciones de negociación colectiva).

Pero, a pesar de compartir propósitos redistributivistas comunes, el giro a la izquierda en América Latina no representó la homogeneidad de un único proyecto de gobernanza a la izquierda. Cada país seguió su ritmo de reformas, presentando gran variedad de intensidad y dirección. La explicación más influyente para esa variedad es de que la sostenibilidad de las políticas implantadas por los gobiernos de izquierda depende del consentimiento de la élite económica, sea doméstica o extranjera, ya que hay una fuerte dependencia del capital para para el mantenimiento de la estabilidad económica y generación de empleos (Przeworski y

Revista Brasileira de Políticas Públicas e Internacionais, v.4, n.1, julho/2019, pp. 58-81. 
CIMINI, Fernanda; AGUIAR, Izabella Leal Miranda. El Giro a la Izquierda en el Uruguay de los Años 2000.

Wallerstein 1988, Boix 2003, Acemoglu y Robinson 2006, Campello 2015). Así que en cada país los gobiernos de izquierda han logrado diferentes tipos de apoyo de las élites económicas. Haggard y Kaufman (1997) sostienen que los presidentes que asumen el poder luego de una crisis económica, encabezada por gobiernos de derecha, tienden a recibir más apoyo político de las élites para gobernar a la izquierda y cuentan con el apoyo popular para adoptar medidas más "duras".

En contraste, los análisis basados en factores institucionalistas priorizan las variables que, de alguna forma, están relacionadas a las trayectorias políticas. En otras palabras, se trata de argumentos que depositan en los dibujos institucionales, en las disputas y elecciones políticas la explicación para los diferentes matices de izquierda. El argumento más influyente en esta línea ha sido lo que considera la institucionalización partidista el punto clave para las diferencias entre radicalización y moderación de las izquierdas latinoamericanas (Levitsky y Roberts 2011, Flores-Macías 2012, Melo 2006, Mainwaring y Torcal 2009, Borsani 2008). En el contexto de institucionalización partidaria, para garantizar la estabilidad democrática, las llamadas izquierdas "profesionales" necesitaban adaptar sus plataformas electorales para conquistar el apoyo no sólo de su constituency, sino también de las élites económicas.

En ese sentido, tanto factores estructurales como institucionalistas contribuyeron para que las izquierdas latinoamericanas asumieran diferentes trayectorias. Actualmente, la crisis vivida por esos gobiernos y la reversión de la agenda llevada a cabo por gobiernos de derecha, han puesto en jaque la capacidad del giro a la izquierda para imprimir cambios profundos en la región. En este escenario, una importante cuestión que se plantea se refiere al legado del giro a la izquierda, en sus múltiples facetas, para la trayectoria política y económica de la región, principalmente en lo que se refiere a la creación de condiciones para el combate a las desigualdades económicas.

Para comprender el cambio en curso con el giro a la izquierda latinoamericana, el trabajo recurre a la noción de coyuntura crítica, tal como se utiliza por la vertiente del institucionalismo histórico. Por coyuntura crítica, se entiende: "a period of significant change, which typically occurs in distinct ways in different countries and which is hypothesized to produce distinct legacies" (Collier y Collier 1991, 29).

Revista Brasileira de Políticas Públicas e Internacionais, v.4, n.1, julho/2019, pp. 58-81. 
CIMINI, Fernanda; AGUIAR, Izabella Leal Miranda. El Giro a la Izquierda en el Uruguay de los Años 2000.

Según Collier y Collier (1991), el concepto de coyuntura crítica contiene tres componentes: (i) el reconocimiento de que hubo un cambio significativo en los casos analizados, (ii) el reconocimiento de que ese cambio ocurrió de formas distintas en diferentes casos, y (iii) una hipótesis explicativa sobre sus consecuencias. Conforme explican los autores, si una coyuntura crítica hipotética no produce el legado esperado, entonces no se puede asegurar que se trata de una coyuntura crítica (Collier y Collier 1991, 30). Los momentos de coyuntura crítica representan oportunidades de cambio de trayectoria, de ruptura de patrones previamente establecidos y de creación de nuevos patrones. No por casualidad, esos momentos pueden configurarse como el marco inicial de nuevos procesos de dependencia de trayectoria.

Para el encuadramiento de un momento político, económico y / o social como "coyuntura crítica", Collier y Collier (1991) proponen los "bloques de construcción" que componen el cuadro analítico de coyunturas críticas, son ellos: (i) las condiciones antecedentes a la ocurrencia de la coyuntura crítica, (ii) el proceso de crisis (o clivaje) que produce la coyuntura crítica, y (iii) el legado, también entendido como los mecanismos de reproducción que garantizan la continuidad de la nueva trayectoria. Los autores llaman la atención sobre la importancia de observar la duración de una coyuntura crítica, el timing en que sucede y la secuencia de eventos que la componen. Esto porque el momento de coyuntura crítica es también un momento de dependencia de trayectoria y, a depender de la duración y de la secuencia de eventos, los cambios pueden rápidamente revertirse por las limitaciones institucionales y estructurales, no dejando legados.

Siguiendo este enfoque, Soifer (2012) propone una distinción entre condiciones permisivas y productivas de coyunturas críticas. Según el autor, condiciones permisivas son condiciones necesarias para "aflojar" limitaciones estructurales, mientras que condiciones productivas serían las condiciones que orientan el proceso de cambio en un contexto específico. Ya las condiciones reproductivas serían los mecanismos que garantizarían la continuidad del cambio, después de las condiciones permisivas y productivas, que provocaron la coyuntura crítica, haber perdido fuerza (Soifer 2012).

A partir de la noción de coyuntura crítica, la principal cuestión que orienta el artículo es analizar si el giro a la izquierda en América Latina puede ser considerado una coyuntura crítica,

Revista Brasileira de Políticas Públicas e Internacionais, v.4, n.1, julho/2019, pp. 58-81. 
CIMINI, Fernanda; AGUIAR, Izabella Leal Miranda. El Giro a la Izquierda en el Uruguay de los Años 2000.

en el sentido de desencadenar nueva trayectoria en el enfrentamiento al status quo de las élites económicas. En conjunto - y para una explotación más profunda de los mecanismos causales se trabajó con el método de process-tracing, método que busca construir explicaciones a partir del análisis de eventos secuenciales y contiguos (Bennet y Checkel 2015). Para ello, proponemos un cuadro analítico para identificar las condiciones permisivas, productivas y reproductivas que influyeron en la elección de los gobernantes de izquierda.

Como condiciones permisivas, apuntamos el desgaste económico vivido por las principales economías latinoamericanas, en el marco de las reformas liberales, que desencadenaron, en muchos casos, la corrosión del Estado de bienestar social y la desindustrialización, llevando al descontento de las clases trabajadoras y a una reconfiguración de las élites económicas.

Como condición productiva, elegimos el contexto de ascensión del partido de izquierda, es decir, la forma como los partidos canalizaron las condiciones desestabilizadoras a su favor. Finalmente, como condición reproductiva, analizamos los impulsos redistributivistas vis-à-vis los acuerdos y enfrentamientos trabados con la élite económica.

Cuadro 1: Giro a la Izquierda como Coyuntura Crítica

\begin{tabular}{|c|c|c|}
\hline 1. Condiciones permisivas & 2. Condiciones productivas & 3.Condiciones reproductivas \\
\hline $\begin{array}{c}\text { Crisis económica } \\
\text { Corrosión del Estado de } \\
\text { bienestar social } \\
\begin{array}{c}\text { Desindustrialización } \\
\text { Conformación de las elites }\end{array}\end{array}$ & $\begin{array}{c}\text { Ascensión del partido de } \\
\text { izquierda: agenda, líderes y }\end{array}$ & $\begin{array}{c}\text { La izquierda en el poder: } \\
\text { acuerdos y enfrentamientos }\end{array}$ \\
apoyo & \\
\hline
\end{tabular}

Fuente: Elaboración propia.

\section{El Giro a la Izquierda como Coyuntura Crítica: El Caso Uruguayo}

Los partidos Blanco y Colorado condujeron la transición neoliberal en Uruguay desde la década de 1980, extendiéndose hasta el año 2004. En este proceso, se abandonaron políticas keynesianas y de bienestar social en favor de la liberalización comercial y financiera. Como consecuencia, estos partidos sufrieron impactos en sus apoyo electoral y grado de legitimación social (Lanzaro 2011).

Revista Brasileira de Políticas Públicas e Internacionais, v.4, n.1, julho/2019, pp. 58-81. 
CIMINI, Fernanda; AGUIAR, Izabella Leal Miranda. El Giro a la Izquierda en el Uruguay de los Años 2000.

\section{Condiciones Permisivas.}

Las condiciones permisivas, en un contexto de coyuntura crítica, señalan un momento de inestabilidad del sistema en análisis. Tal estado es oriundo de la flexibilización de restricciones institucionales y estructurales al modelado del status quo, vía actuación de agencias individuales y contingencias mundanas. En este sentido, en el marco del giro a la izquierda en Uruguay, actuaron la crisis económica de 2002, la corrosión del Estado de bienestar social, el proceso de desindustrialización y la fragmentación de las élites.

\subsection{Crisis Económica}

Uruguay pasó por un período de severa crisis económico-financiera, que se extendió desde 1998 hasta principios del año 2003 - alcanzando su punto culminante en el año 2002 muy en virtud de las políticas económicas engendradas por la ola neoliberal mediatizada por el Consenso de Washington. El PIB uruguayo cayó del 3\% en 1999 al 1\% en 2000. Más allá, en el año 2000, la inflación anual subió a casi el 6\% y la tasa de desempleo superó el 13\%; hubo desaceleración del crédito al consumidor; el salario real se deterioró, retrocediendo en más del $1 \%$ y el déficit del sector público superó el 4\% del PIB (Comisión Económica para América Latina y el Caribe [CEPAL] 2000).

El escenario externo, que había sido ampliamente favorable para el país durante la mayor parte de los años noventa, estaba en proceso de retracción. En el año 2000, se registró caída de las exportaciones a Brasil y Argentina, a pesar del crecimiento de la economía brasileña. Con el aumento del precio del petróleo y la disminución del precio de las exportaciones uruguayas, se formó un déficit en la cuenta corriente del balance de pagos del $3 \%$, financiado con endeudamiento externo. Más allá, los trastornos climáticos y el resurgimiento de la fiebre aftosa en el año 2000 perjudicaron a la agropecuaria (CEPAL 2000).

Cuando la crisis alcanza su punto culminante, en 2002, el PIB sufre una caída del 10,5\%; la inflación acelera al 25\% anual; los salarios reales sufren una caída superior al 10\% y la tasa de desempleo alcanza el 19\% - un pico histórico, abarcando casi una quinta parte de la población económicamente activa. En este año, las exportaciones uruguayas cayeron un 10\% -

Revista Brasileira de Políticas Públicas e Internacionais, v.4, n.1, julho/2019, pp. 58-81. 
CIMINI, Fernanda; AGUIAR, Izabella Leal Miranda. El Giro a la Izquierda en el Uruguay de los Años 2000.

para Argentina, esa cifra alcanza el 65\% - y las importaciones cayeron un 30\%, en virtud de la devaluación del peso uruguayo y de la caída de la demanda interna (CEPAL 2002).

La crisis financiera argentina tuvo gran impacto sobre Uruguay, que sufrió con una retirada masiva de los depósitos de no residentes. Las reservas internacionales del país sufrieron caída y Uruguay perdió su calificación de investment grade que le había permitido obtener financiamiento externo a bajas tasas. En respuesta, el Banco Central sustituye el sistema de bandas deslizantes por el de cambio flotante, creando mayor inseguridad en un escenario de cambio volátil. Como resultado, la moneda uruguaya se desvalorizó, haciendo crecer aún más el endeudamiento de los sectores público y privado en dólares. Sin embargo, la devaluación del peso argentino fue aún mayor, causando la pérdida de competitividad externa por parte de Uruguay (CEPAL 2002).

En este año, se observó un descenso de la demanda, tanto interna como externa, desalentando la industria manufacturera, la construcción y los servicios de comercio, con una caída de más del 10\% en todos ellos. Con el deterioro de la recaudación tributaria, se mantuvo un alto déficit fiscal (CEPAL 2002).

Tabla 1: Tasas de Variación Anuales del PIB y de la Inflación en Uruguay 2000-2002 (En Porcentaje)

\begin{tabular}{|c|c|c|c|}
\cline { 2 - 4 } \multicolumn{1}{c|}{} & 2000 & 2001 & 2002 \\
\hline Producto Interno Bruto & $-1,9$ & $-3,4$ & $-10,5$ \\
\hline Precios al consumidor & 5,1 & 3,6 & 24,7 \\
\hline
\end{tabular}

Fuente: Extraído de CEPAL (2002, p. 71).

Como consecuencia de este proceso de continuo deterioro económico, bajo la égida de gobiernos de inspiración neoliberal, se profesó, entre la población uruguaya, un sentimiento común de insatisfacción frente a la ideología y práctica asumidas por los gobiernos de entonces. La postura pro mercado, en este período, era inmediatamente relacionada al fracaso económico y a la escasez en el medio social. Fue la crisis económica, por lo tanto, una condición permisiva para el fortalecimiento de las agremiaciones políticas a la izquierda: estos grupos proponían una agenda alternativa, socialmente preocupada.

Revista Brasileira de Políticas Públicas e Internacionais, v.4, n.1, julho/2019, pp. 58-81. 
CIMINI, Fernanda; AGUIAR, Izabella Leal Miranda. El Giro a la Izquierda en el Uruguay de los Años 2000.

Tabla 2: Satisfacción Uruguaya con la Economía de Mercado

\begin{tabular}{|c|c|c|c|c|c|}
\hline iLa economía de mercado & \multicolumn{5}{|c|}{ URUGUAY } \\
\cline { 2 - 6 } es lo mejor para el país? & $\mathbf{1 9 9 8}$ & $\mathbf{2 0 0 0}$ & $\mathbf{2 0 0 2}$ & $\mathbf{2 0 0 7}$ & $\mathbf{2 0 0 9}$ \\
\hline Muy de acuerdo & 13 & 10 & 6 & 10 & 13 \\
\hline De acuerdo & 47 & 45 & 45 & 65 & 65 \\
\hline En desacuerdo & 27 & 24 & 30 & 22 & 18 \\
\hline Muy en desacuerdo & 14 & 21 & 19 & 3 & 5 \\
\hline$(\mathbf{N})$ & $\mathbf{8 9 8}$ & $\mathbf{8 2 7}$ & $\mathbf{8 0 8}$ & $\mathbf{7 9 2}$ & $\mathbf{8 5 5}$ \\
\hline
\end{tabular}

Fuente: Extraído de Latinobarómetro.

\subsection{Corrosión del Estado de bienestar social}

En lo que concierne a la corrosión del Estado de bienestar, se identifica, durante los gobiernos neoliberales, una miríada de cuestiones sociales puestas en segundo plano o sumariamente ignoradas.

Durante la crisis de 2001, el seguro de desempleo (con cobertura de seis meses) sólo alcanzó el $17 \%$ de los desempleados, resultando en un flujo migratorio de población económicamente activa al extranjero (De Dios 2015). Concomitantemente, se observó la pérdida de autonomía de los sindicatos - actores colectivos estratégicos para reclutamiento y movilización electoral - especialmente en el ámbito de las relaciones laborales en la iniciativa privada. Los sindicatos, que habían sido muy importantes para la transición democrática (198085), sufrieron con la transición neoliberal que se siguió, perdiendo miembros, densidad y poder de negociación. No obstante, estos grupos conservaron su federación nacional y se opusieron a las reformas de mercado, en especial a las privatizaciones (Lanzaro 2011).

La pobreza dobló en Uruguay como resultado de la crisis de 2001, en conjunto con las malogradas políticas del gobierno que, una vez más dando muestras de su total divorcio con el principio redistributivo, actuó aumentando los impuestos indirectos sobre el consumo por medio del Impuesto al Valor Agregado (IVA) (Lanzaro 2011). En el campo, se profundizaron los problemas sociales con la desaparición de casi el 30\% del total de establecimientos familiares y el incremento de las desigualdades: la mitad de los trabajadores rurales y agricultores familiares vivieron en situación de pobreza (Riella y Andrioli 2004).

El avance desregulador y privatizador, moderado por un plebiscito de 1992, en que la población rechazó la privatización de empresas públicas, fue retomado con vigor en 1999, a

Revista Brasileira de Políticas Públicas e Internacionais, v.4, n.1, julho/2019, pp. 58-81. 
CIMINI, Fernanda; AGUIAR, Izabella Leal Miranda. El Giro a la Izquierda en el Uruguay de los Años 2000.

pesar de la expresión popular contraria: se trata de gobiernos cuyos movimientos, a los ojos de expresiva parte de población, se veían ilegítimos (Bittencourt 2006).

Con prácticas políticas contrarias al mantenimiento del Estado de bienestar social, los gobiernos neoliberales cargaban dentro de sí la semilla de su propia destrucción: la sujeción de la población a arduas condiciones de vida acabó por unirla y la hizo erigir junta, abierta a nuevas banderas e ideales. Se hizo más una condición permisiva para la ascensión de la izquierda.

\subsection{Desindustrialización}

El proceso de desindustrialización reciente en Uruguay se remonta a finales de los años 80, período en que ya se había cerrado la mitad de las empresas en el sector (Rilla 2008). La vigencia del modelo económico neoliberal en los años noventa resultó en fracaso en términos de mejora del crecimiento a largo plazo de Uruguay. Tal modelo propugnó la liberalización comercial y financiera, con abandono del proteccionismo en favor de una adopción sistemática de la ley de las ventajas comparativas. Con eso, el país fue inducido a una especialización basada en productos primarios, de manera tal a generar mayor volatilidad - una vez sujeto a oscilaciones de la demanda del mercado mundial por bienes primarios - desaguando, por fin, en problemas relacionados a la generación de empleos y distribución de la renta. En lo que concierne a estas consecuencias, se sabe que el aumento del desempleo ya era vigente antes de la recesión de 1999 y de la crisis de 2002, siendo por lo tanto reflejo directo de las políticas neoliberales.

Las políticas comerciales y cambiarias en este período motivaron, de esta forma, la desindustrialización de la economía - la participación de la industria en el PIB fue del 29\% en 1985, cayendo al 17\% en 1999-2001 - habiendo la agropecuaria aumentado significativamente su producción (Bittencourt 2006).

En el ínterin, los procesos de privatización de las grandes empresas condujeron al aumento de la Inversión Extranjera Directa (IED) en Uruguay. La IED, sin embargo, se dirigió a sectores primarios y a sectores industriales intensivos en recursos naturales - habiendo reducción de los capitales destinados a sectores relacionados a la sustitución de importaciones al contrario de lo que se podría pensar, al suponerse un mayor potencial de diversificación

Revista Brasileira de Políticas Públicas e Internacionais, v.4, n.1, julho/2019, pp. 58-81. 
CIMINI, Fernanda; AGUIAR, Izabella Leal Miranda. El Giro a la Izquierda en el Uruguay de los Años 2000.

productiva con la ampliación del mercado oriunda de la influencia de las empresas transnacionales (Bittencourt et al. 2009).

Como corolario, se constató una fuerte concentración de las exportaciones en pocos sectores cercanos a la base primaria, caracterizados por un bajo dinamismo internacional y centralización en pocas grandes empresas. La producción de bienes con mayor valor agregado y alto contenido tecnológico, a su vez, se limitó a cerca de 1/10 de la producción del país (industria de escala, como la automotriz y de medicamentos / químicos) (Bittencourt et al. 2009).

Se observa, por medio de esta dinámica, que el flujo de capitales hacia el país profundizó los procesos de primarización del patrón de especialización comercial y de desindustrialización. Más allá, este flujo de capitales contribuyó para el financiamiento de déficits oriundos de la diferencia entre la importación de bienes intensivos en tecnologías y la exportación de bienes primarios, evidenciando la sumisión uruguaya a la estructura económica internacional (Bittencourt et al. 2009).

La ralentización industrial, junto a la crisis económico-financiera de 2002, afectaron la credibilidad del gobierno neoliberal y del subsistema partidista tradicional que lo sustentó por más de un siglo, instalando en las élites y en la población la convicción de que se había saturado el entonces vigente modelo político-económico (Rilla 2008).

\subsection{Conformación de las Élites}

Con su génesis en el siglo XIX, los partidos Blanco (Nacional) y Colorado formaron el más tradicional arreglo político uruguayo, extendiendo su influencia desde el período del nacimiento de la República hasta la ascensión del partido Frente Amplio al poder, en 2005. En el espectro de conformación ideológica, el partido Blanco tiende a una centro derecha relativamente hermética, asociada a una oligarquía rural, mientras que el partido Colorado se inclina hacia una centro derecha comprometida con el desarrollo liberal del mercado y más

Revista Brasileira de Políticas Públicas e Internacionais, v.4, n.1, julho/2019, pp. 58-81. 
CIMINI, Fernanda; AGUIAR, Izabella Leal Miranda. El Giro a la Izquierda en el Uruguay de los Años 2000.

abierta a concepciones socialmente preocupadas, teniendo la influencia del batllismo ${ }^{1}$ en su formación. La travesía de dos siglos, sin embargo, arraigó prácticas e institucionalizó costumbres políticas en estos partidos que se mostraron incompatibles con la dinámica políticoeconómica del siglo XXI.

El poder económico, que antes gravitaba alrededor de élites conservadoras asociadas a los partidos tradicionales, sufrió desplazamiento frente a los cambios institucionales emprendidos a partir de finales del siglo XX. La práctica política del clientelismo sufrió corrosión junto al clásico reclutamiento de empresarios vía capital familiar heredado y al prestigio de la propiedad de la tierra - en virtud de la creciente influencia del capital financiero internacional - amenazando terminantemente la estructura oligárquica de estos partidos (Luna 2007). Estos cambios se hicieron en favor de un nuevo modelo de control y gestión de empresas profesionalizado en redes globales y nuevas formas de capital social, con reinvención institucional constante, especialización flexible de la producción y redes menos jerárquicas (Serna 2013).

Reside en esta dinámica más una condición permisiva al ascenso de la izquierda en Uruguay: la falta de resiliencia de los partidos tradicionales frente a los cambios institucionales del siglo XXI hizo menos combativa su oposición a la subida del Frente Amplio al poder.

En lo que concierne a la cohesión de la élite uruguaya, se evidencian puntos de profunda fricción en las relaciones que se establecen en el seno de esa clase. Dentro de este sistema, se observa una miríada de cámaras industriales y mercantiles que, al impedir la convergencia de los intereses empresariales en una única organización colectiva, dificulta la formulación de estrategias conjuntas, lo que produce demandas heterogéneas y políticamente poco robustas (Diniz 2016).

En lo que concierne al carácter fragmentado asumido por la élite uruguaya, es de destaque la posición tomada por la elite pecuaria - sector de destacada influencia en la economía - frente a élites pertenecientes a otras ramas de la economía uruguaya.

\footnotetext{
${ }^{1}$ Corriente del Partido Colorado creada por José Batlle y Ordóñez - presidente de la República en los períodos de 1903 a 1907, y 1911 a 1915 - que propugna el intervencionismo estatal en favor del desarrollo económico y del bienestar social.
}

Revista Brasileira de Políticas Públicas e Internacionais, v.4, n.1, julho/2019, pp. 58-81. 
CIMINI, Fernanda; AGUIAR, Izabella Leal Miranda. El Giro a la Izquierda en el Uruguay de los Años 2000.

Aunque la imagen del latifundista afecto a las prácticas locales tradicionales de dominación social se haya diluido en favor de aquella del empresario rural preocupado por la rentabilidad de su negocio, en el siglo XXI, todavía se identifica una posición marcadamente reaccionaria entre la élite pecuaria (Riella y Andrioli 2004).

Guiados por una mentalidad eminentemente fisiocrática, los ganaderos proponen sus formas de apropiación y uso de la tierra como las únicas posibles y legítimas: cualquier otra propuesta iría contra el orden natural de las cosas, siendo irresponsable e irracional. La génesis del papel social pecuario está en su actuación en conformidad con las condiciones naturales del país: el sector de servicios, por ejemplo, es visto como una moda pasajera frente a la esencial vocación agropecuaria (Riella y Andrioli 2004).

Los ganaderos presentan sus intereses particulares como intereses generales de la nación, haciendo su legitimación vía capital simbólico - por medio del mito del país pecuarista, se propone que el único destino posible para Uruguay sea la producción de carne y lana. De esta forma, la pecuaria se auto-consagra jerárquicamente superior a las demás actividades del campo, de manera tal a suscitar una desunión con la - también fuerte - élite agrícola. Más allá de la agropecuaria, la élite pecuaria se ve en conflicto con el capital financiero y la industria frigorífica: los considera estériles en sus rasgos especulativos y burocráticos, a pesar de tratarse de sectores en constante proceso de innovación y dotados de mayor dinamismo económico (Riella y Andrioli 2004).

La desunión de las élites nacionales se suma al conflicto de intereses con la élite internacional, que toma importantes espacios en la economía uruguaya. Es evidente la existencia de un creciente control por parte del capital extranjero de los sectores exportadores clave: a comienzos de los años 2000, llegó a representar el 6\% del PIB, 1/3 del total de inversión de la economía y el 40\% de la inversión privada en el país (Bittencourt et al. 2009).

En vista de la conformación de la élite uruguaya en el período anterior al giro a la izquierda, se puede concluir que su limitada cohesión y, por lo tanto, su bajo poder de movilización, contribuyó para que el partido Frente Amplio ampliase su espacio de actuación y conquistase apoyo de los sectores insatisfechos de la élite uruguaya, colocándose como una condición permisiva a tal ocurrencia.

Revista Brasileira de Políticas Públicas e Internacionais, v.4, n.1, julho/2019, pp. 58-81. 
CIMINI, Fernanda; AGUIAR, Izabella Leal Miranda. El Giro a la Izquierda en el Uruguay de los Años 2000.

\section{Condiciones Productivas}

Las condiciones productivas se erigen sobre circunstancias previamente modeladas por las condiciones permisivas. La acción productiva es ordenadora: canaliza los núcleos de inestabilidad del sistema hacia una ocurrencia de naturaleza específica. A partir de esta convergencia de fuerzas dispersas, se concreta el cambio. En este sentido, las estrategias de campaña del partido Frente Amplio canalizaron eficazmente las insatisfacciones oriundas de las condiciones desestabilizadoras anteriormente abordadas, a su favor.

\subsection{Ascensión del Frente Amplio al poder: agenda, líderes y apoyo}

El Frente Amplio reposó su campaña en la promesa de superación profunda de los problemas económicos y sociales acumulados a lo largo de las administraciones neoliberales, explorando la posibilidad de reversión del "pesimismo estructural" que se había instalado en el país (Luna 2002).

Tabla 3: Evolución de las Evaluaciones sobre Situación Económica

\begin{tabular}{|l|c|c|c|}
\hline $\begin{array}{l}\text { ¿Cómo calificaria Ud. la situación económica actual del } \\
\text { país? }\end{array}$ & 1994 & 1999 & 2004 \\
\hline Muy buena & 1 & - & - \\
\hline Buena & 12 & 6 & 6 \\
\hline Ni buena ni mala & 39 & 26 & 25 \\
\hline Mala & 33 & 47 & 36 \\
\hline Muy mala & 15 & 21 & 33 \\
\hline Total & 100 & 100 & 100 \\
\hline
\end{tabular}

Fuente: Extraído de Queirolo (2006, p. 43) a partir de investigación preelectoral hecha por la consultora CIFRA.

Los gobiernos anteriores no pudieron establecer acuerdos favorables a todos los segmentos sociales - beneficiando tan sólo a los segmentos históricamente acumuladores de capital - y hubo falta de diálogo con la sociedad civil - lo que habría impedido la cooperación entre capital y trabajo. Por el contrario, el Frente Amplio se dispuso a emprender una política conciliatoria entre capital y trabajo por medio de un abordaje concomitantemente neodesarrollista y neocorporativista. Se propuso un modelo en el que la frontera que separa al

Revista Brasileira de Políticas Públicas e Internacionais, v.4, n.1, julho/2019, pp. 58-81. 
CIMINI, Fernanda; AGUIAR, Izabella Leal Miranda. El Giro a la Izquierda en el Uruguay de los Años 2000.

económico del social - capital del trabajo - se redujera al punto que el favorecimiento de uno de ellos fuera igualmente revertido para el otro. En suma, el gobierno Frente Amplio se mostró visiblemente comprometido con la edificación de una coexistencia sana entre el mercado y políticas sociales universalistas (Busnello 2006).

Más allá, en su proyecto de recuperación económica, el gobierno declaró apoyo total a los sectores productivos con incentivos fiscales - reducción gradual de las cargas patronales a la seguridad social - e inversiones públicas - dirigidas a sectores de la producción más competitivos, con mayor potencial de generación de empleo y de desarrollo tecnológico, con el objetivo de aumentar la producción, disminuir el desempleo, aumentar los salarios y mejorar la distribución de renta, además de insertar a Uruguay de manera competitiva y soberana en el mundo (Busnello 2006).

Con el fin de atribuir mayor grado de solidez a su compromiso con una política de izquierda relativamente moderada frente al libre mercado, Tabaré Vázquez anunció su intención de nombrar a Danilo Astori como Ministro de Economía, conocido defensor de las inversiones privadas y de la competencia de mercado. Al mismo tiempo, el candidato impone fuerza simbólica a su personalidad al oponerse, junto a la mayoría de la población uruguaya en referéndum, a la propuesta de privatización de las empresas de tratamiento y distribución de agua potable por parte del gobierno neoliberal (Busnello 2006).

De esta forma, sin levantar polémicas o indicar una ruptura total con el modelo políticoeconómico entonces vigente, las estrategias de campaña adoptadas por el Frente Amplio aspiraban a una doctrina del medio-término aristotélico, es decir, la virtud necesaria para la superación de la crisis estaría en el equilibrio: “(...) la alianza constituida en torno a la candidatura de Tabaré Vázquez supo explotar la situación de crisis que Uruguay enfrentaba en el momento de la elección presidencial, para indicar la necesidad de un cambio político que fuera más allá del bipartidismo hasta entonces vigente.” (Busnello 2006, 28).

Esta tercera vía, a un tiempo pragmática y progresista, se puso en sintonía con una fracción del electorado de creciente peso demográfico en Uruguay: los jóvenes. La juventud uruguaya es fuertemente inclinada hacia el apoyo a la izquierda frenteamplista, colocándose

Revista Brasileira de Políticas Públicas e Internacionais, v.4, n.1, julho/2019, pp. 58-81. 
CIMINI, Fernanda; AGUIAR, Izabella Leal Miranda. El Giro a la Izquierda en el Uruguay de los Años 2000.

como agente estratégico de movilización social en favor del partido (Aguiar 2000 apud Buquet y Chasquetti 2005).

A partir de una campaña capaz de complacer los intereses, concomitantemente, de las masas populares (incluidos los jóvenes), esperanzadoras frente a la posibilidad de fortalecimiento del Estado de bienestar social, y de las élites económicas, seguras frente a la agenda pro mercado, el Frente Amplio logró imponerse como alternativa viable a la crisis que vivía el país.

En las elecciones de octubre de 2004, el Frente Amplio alcanza la Presidencia de la República sin necesidad de segunda vuelta, obteniendo el 50,5\% del total de votos y la mayoría parlamentaria en las dos cámaras (senadores y diputados).

\section{Condiciones de Reproducción}

El mecanismo de reproducción es lo que permite que el cambio se prolongue a través del tiempo y, por consiguiente, viabiliza su extensión en el espacio. Se trata, pues, de la capacidad del cambio de perdurar frente a la agencia y a la contingencia. En este sentido, el Frente Amplio adoptó estrategias políticas resilientes y pudo contar con la contingencia a su favor, de manera tal a reproducir la ocurrencia del giro a la izquierda.

\subsection{Frente Amplio: contingencia, políticas y resiliencia}

Con la victoria de Tabaré Vázquez del partido Frente Amplio en las elecciones presidenciales de 2004, la izquierda experimentó por primera vez en la historia uruguaya, su ascenso al puesto más alto del país.

Para la sustentación de su posición, el Frente Amplio contó con algunos eventos que escaparía a su alzada: se puede decir que la Virtù vino debidamente acompañada de su Fortuna. En este caso, tanto la agencia como la contingencia trabajaron como mecanismos de reproducción.

A comienzos de los años 2000, se produjo un ascenso en el precio de los commodities, que se extendió hasta el año 2008, reavivando el mercado interior y el empleo, incluso frente a una subida del precio del petróleo (Lanzaro 2011).

Revista Brasileira de Políticas Públicas e Internacionais, v.4, n.1, julho/2019, pp. 58-81. 
CIMINI, Fernanda; AGUIAR, Izabella Leal Miranda. El Giro a la Izquierda en el Uruguay de los Años 2000.

Ya con el fenómeno del bono demográfico - composición de la pirámide de edad que presenta una proporción de población económicamente activa relativamente superior a las poblaciones económicamente dependientes, es decir, niños y ancianos - hubo un aumento en el número de contribuyentes, de manera tal a incrementar las ganancias del gobierno (De Dios 2015).

Consciente de la importancia de los apoyos concedidos por la élite industrial y los sindicatos, el gobierno los unió en los Consejos de Salarios ${ }^{2}$, con el fin de hacer deliberaciones concernientes a la actividad productiva: este nuevo pacto social fue de suma importancia para el avance de la industrialización en el país. Ya en 2005, se percibía un aumento en la participación de la industria en el PIB, que pasó del 17\% en 1999-2001 al 22\% en el primer año de gobierno (Bittencourt 2006), contribuyendo al alcance de tal cifra el fomento de la Corporación Nacional para el Desarrollo - Banco Público de Inversión uruguayo. En este proceso, el Estado estuvo siempre al frente, de manera tal a indicar un mantenimiento significativo de las capacidades estatales incluso después de las iniciativas corrosivas de los gobiernos liberales contra su aparato.

Como se indica en la tabla II, hubo una evolución significativa en los activos e inversiones por parte de los principales Bancos Públicos de Inversión de Uruguay - Banco de la República Oriental del Uruguay, Banco Hipotecario del Uruguay y Corporación Nacional para el Desarrollo - tras la ascensión del Frente Amplio, lo que evidencia el apoyo estatal a estas instituciones (De Olloqui y Palma 2013).

El gobierno se comprometió con las leyes del mercado y con el mantenimiento de la estabilidad, lanzando mano de cierto grado de ortodoxia - tipo de cambio estable y control del déficit fiscal - lo que aseguró las condiciones para la acción privada. En el marco de este proceso, se verificó un aumento en la Inversión Extranjera Directa, incentivo a la inversión privada en el sector público, de servicios, infraestructura y agroindustria y promoción del comercio y de la competitividad por vía legislativa (Lanzaro 2011).

\footnotetext{
2 Áreas de negociación tripartita - Poder Ejecutivo, empresarios y trabajadores - para deliberar cuestiones concernientes a la política salarial.
}

Revista Brasileira de Políticas Públicas e Internacionais, v.4, n.1, julho/2019, pp. 58-81. 
CIMINI, Fernanda; AGUIAR, Izabella Leal Miranda. El Giro a la Izquierda en el Uruguay de los Años 2000.

Tabla 4: Evolución de los Activos e Inversiones de los Bancos Públicos de Desarrollo en Uruguay (En Porcentaje)

\begin{tabular}{|c|c|}
\hline $\begin{array}{c}\text { Activos de los Bancos Públicos de } \\
\text { Desarrollo }\end{array}$ & $\begin{array}{c}\text { Colocaciones de los Bancos Públicos de } \\
\text { Desarrollo }\end{array}$ \\
\hline Variación Promedio Anual Período 2000-05 \\
\hline$-5,8$ & $-7,3$ \\
\hline \multicolumn{2}{|c|}{ Variación Promedio Anual Período 2005-10 } \\
\hline 12,6 & 15,2 \\
\hline
\end{tabular}

Fuente: Extraído de De Olloqui y Palma (2013, p. 3), basados en Databank.

En el plano social, el Frente Amplio exhibe su fuerza al aprobar proyectos de ayuda universal, en vez de aquellos restringidos a sólo determinado segmento de la población. En este sentido, figuran las reformas sanitaria, tributaria, contra la pobreza (PANES, Plan de Equidad), leyes para la legalización del matrimonio homoafectivo, de la marihuana, del aborto y de la eutanasia (Pribble y Huber 2011).

Su primera iniciativa fue la introducción de un plan de emergencia social, el PANES, un programa de transferencias para familias de bajos ingresos - sustituido en 2007 por el Plan de Equidad - que institucionalizó la ayuda a familias de bajos ingresos, redujo la edad de acceso al sistema de pensiones no contributivas y vinculó el plan a la escolarización de los niños (De Dios 2015).

En 2007, el Frente Amplio reformó el sistema de salud para mejorar el servicio a través del Sistema Nacional Integrado de Salud: el sistema ofrece una protección a la salud casi universal con servicios de la misma calidad a todos los ciudadanos. En el ámbito educativo, el porcentaje del PIB destinado a la educación pasó del 3,6\% en 2001 al 5,3\% en 2011 (De Dios 2015).

La reforma fiscal del gobierno tuvo como objetivo viabilizar una redistribución de ingresos más equitativa. Para ello, se puso en marcha la disminución de los impuestos indirectos - sobre el comercio - y el aumento de los impuestos directos, creándose un verdadero impuesto sobre la renta: éste no se limitó a salarios o pensiones, extendiéndose a todos los tipos de ingresos de personas físicas (Lanzaro 2011).

Al hacerse un análisis global del país, se evidencia el efecto del impulso redistributivo de renta dado por el Frente Amplio. Uruguay, aunque sea un país pequeño, registra

Revista Brasileira de Políticas Públicas e Internacionais, v.4, n.1, julho/2019, pp. 58-81. 
CIMINI, Fernanda; AGUIAR, Izabella Leal Miranda. El Giro a la Izquierda en el Uruguay de los Años 2000.

históricamente importantes divergencias entre los departamentos nacionales: aquellos al norte y noroeste son menos favorecidos económicamente, mientras que los de la franja sur (próximos a Montevideo) presentan altos niveles de renta. Aunque la Constitución de 1996 tenga una orientación potencialmente descentralizadora, estos cambios muchas veces no se materializaron. Como se muestra en la figura 1, la desigualdad regional se hizo creciente de la década de 1990 hasta los años 2000. A partir de los años 2000, sin embargo, hubo una tendencia a la ecualización de las rentas entre los departamentos (Muinelo-Gallo y Miranda 2014).

Figura 1: Relación entre la Renta per Cápita de las Familias en cada Departamento y el Valor Medio de Montevideo
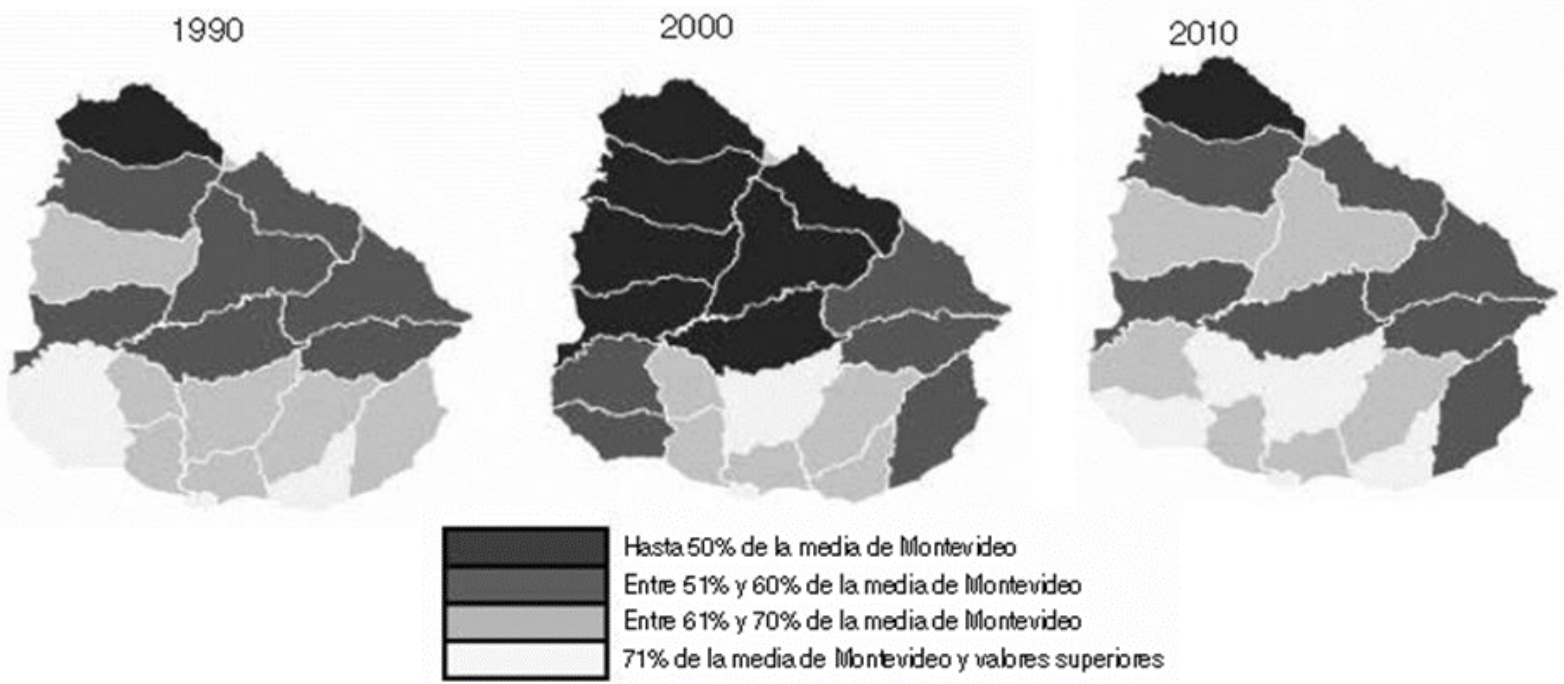

Fuente: Extraído de Muinelo-Gallo y Miranda (2014, 221), con datos del Instituto Nacional de Estadística de Uruguay.

Gracias a las políticas del Frente Amplio, Uruguay representa actualmente un modelo de desarrollo de Estado de bienestar universalista (institucional-redistributivo) en América Latina (De Dios 2015).

Por fin, a través del análisis de las estrategias políticas adoptadas por el Frente Amplio, resulta evidente que la piedra de toque de su estabilidad - su mecanismo de reproducción reside en su resiliencia frente a las demandas de la élite y de los trabajadores: el emprendimiento de un modelo político de coexistencia entre mercado y políticas sociales

Revista Brasileira de Políticas Públicas e Internacionais, v.4, n.1, julho/2019, pp. 58-81. 
CIMINI, Fernanda; AGUIAR, Izabella Leal Miranda. El Giro a la Izquierda en el Uruguay de los Años 2000.

universalistas fue el combustible que permitió al gobierno de izquierda uruguayo prolongarse en el tiempo ${ }^{3}$ y materializarse en el espacio.

\section{Consideraciones Finales}

La investigación emprendida trajo respuestas a las preguntas inicialmente elaboradas y suscitó nuevas preguntas. Al analizarse la coyuntura crítica del giro a la izquierda en Uruguay, se verificó la influencia directa del período neoliberal anterior en la conformación de las capacidades estatales del país. Se identificó una destacada limitación en la corrosión del aparato estatal durante los gobiernos neoliberales: éstos no fueron capaces de poner en marcha el núcleo de sus programas liberalizantes. De esta forma, el Estado en Uruguay probó ser una institución sólida, deteniendo una histórica inclinación a la coordinación de políticas universalistas de bienestar social.

En lo que concierne a la configuración de las élites, se atestiguó su naturaleza fragmentaria: el conflicto de intereses, especialmente en el plano agro versus industria, perjudica profundamente la cohesión de este grupo social. Como corolario, se verificó el desprendimiento de la élite industrial y su asociación al gobierno de izquierda, en detrimento de la élite agropecuaria.

Más allá, la política conciliatoria adoptada por la izquierda uruguaya en lo que se refiere al mercado - especialmente en el ámbito de la IED - acabó por permitir la prosecución, aunque de forma menos exacerbada, del proceso de primarización de la economía del país. Tal tendencia se consolida a través del fortalecimiento de sectores industriales intensivos en recursos naturales que, con una producción de bajo valor agregado, limitado potencial innovador y reducidos aumentos de productividad, acaba por limitar la propia magnitud de los recursos disponibles para las políticas de bienestar social - uno de los principales pilares sostenedores del gobierno Frente Amplio.

Esta postura gubernamental, por lo tanto, no sólo perjudica las tasas de crecimiento del país a largo plazo, como amenaza la continuidad de las exitosas políticas de bienestar social

\footnotetext{
${ }^{3}$ El partido Frente Amplio está al frente del gobierno uruguayo desde las elecciones de 2004 hasta el momento en que se escribe este artículo.
}

Revista Brasileira de Políticas Públicas e Internacionais, v.4, n.1, julho/2019, pp. 58-81. 
CIMINI, Fernanda; AGUIAR, Izabella Leal Miranda. El Giro a la Izquierda en el Uruguay de los Años 2000.

emprendidas, en el caso de que no haya un profundo proceso de redistribución equitativa de los recursos nacionales.

\section{Referencias Bibliográficas}

Acemoglu, Daron y James A. Robinson. 2006. Economic Origins of Dictatorship and Democracy. New York: Cambridge University Press.

Ansell, Ben y David Samuels. 2014. Inequality and Democratization: An Elite-Competition Approach. Cambridge: Cambridge University Press.

Bennett, Andrew y Jeffrey T. Checkel (eds.). 2015. Process Tracing: From Metaphor to Analytic Tool (Strategies for Social Inquiry). Cambridge: Cambridge University Press.

Bittencourt, Gustavo. 2006. "Uruguay 2006: desarrollo esquivo o ruptura con la historia". América Latina Hoy 44: 15-39. http://www.redalyc.org/pdf/308/30804402.pdf

Bittencourt, Gustavo, Gastón Carracelas, Andrea Doneschi y Nicolás Reig Lorenzi. 2009. Tendencias Recientes de la Inversión Extranjera Directa en Uruguay. Montevidéu: Universidad de la República.

Boix, Carles. 2003. Democracy and Redistribution. New York: Cambridge University Press.

Buquet, Daniel y Daniel Chasquetti. 2005. "Elecciones Uruguay 2004: descifrando el cambio". Revista de Ciencia Política 25(2): 143-152. http://dx.doi.org/10.4067/S0718090X2005000200006

Busnello, Flávio Marcelo. 2006. "Uma nova esquerda? A tensão entre capital e trabalho nas candidaturas de Luiz Inácio Lula da Silva (Brasil, 2002) e de Tabaré Vázquez (Uruguai, 2004)”, tesis de maestría, Universidade de Brasília, Brasil.

Campello, Daniela. 2015. The Politics of Market Discipline in Latin America: Globalization and Democracy. Cambridge: Cambridge University Press.

Collier, Ruth Berins y David Collier. 1991. Shaping the political arena. Princeton: Princeton University Press.

Comisión Económica para América Latina y el Caribe (CEPAL). 2000. Balance Preliminar de las economías de América Latina y el Caribe, 2000. Santiago: CEPAL.

Revista Brasileira de Políticas Públicas e Internacionais, v.4, n.1, julho/2019, pp. 58-81. 
CIMINI, Fernanda; AGUIAR, Izabella Leal Miranda. El Giro a la Izquierda en el Uruguay de los Años 2000.

Comisión Económica para América Latina y el Caribe (CEPAL). 2002. Balance Preliminar de las economías de América Latina y el Caribe, 2002. Santiago: CEPAL.

De Dios, Manuel Sánchez. 2015. "La Reforma de los Regímenes de Bienestar en Costa Rica, Chile, Argentina, Brasil y Uruguay”. Política y Sociedad 52 (3): 661-690. http://dx.doi.org/10.5209/rev_POSO.2015.v52.n3.45377

De Olloqui, Fernando y Cristian Palma. 2013. "Panorama y evolución reciente de los bancos públicos de desarrollo". En Bancos Públicos de Desarrollo ¿Hacia un Nuevo Paradigma? Editado por Fernando de Olloqui, 1-20. Washington, D. C.: Banco Interamericano de Desarrollo.

Diniz, Eli. 2013. "Desenvolvimento e Estado Desenvolvimentista: tensões e desafios da construção de um novo modelo para o Brasil do Século XXI". Revista de Sociologia e Política, 21 (47): 133-153. http://dx.doi.org/10.1590/S0104-44782013000300002.

Diniz, Eli. 2016. "Empresariado e projeto neoliberal na América Latina: uma avaliação dos anos 80". En Estado e Sociedade no Brasil: a obra de Renato Boschi e Eli Diniz. Editado por José Szwako, Rafael Moura y Paulo D’Avila Filho, 349-377. Rio de Janeiro: Instituto Nacional de Ciência e Tecnologia em Políticas Públicas, Estratégias e Desenvolvimento.

Fairfield, Tasha. 2015. Private Wealth and Public Revenue: Business Power and Tax Politics in Latin America. Cambridge: Cambridge University Press.

Filgueira, Fernando. 2013. "Los Regímenes de Bienestar en el caso de la modernización conservadora: posibilidade y lìmites de la ciudananía social en America Latina". Revista $\begin{array}{lllll}\text { Uruguaya de } & \text { Ciencia } & \text { Política } & \text { 22(2): }\end{array}$ http://www.redalyc.org/articulo.oa?id=297330013002

Fioretos, Orfeo, Tulia G. Falleti y Adam Sheingate. 2016. The Oxford Handbook of Historical Institutionalism. Oxford: Oxford University Press.

Flores-Macias, Gustavo. 2012. After Neoliberalism? The Left and Economic Reforms in Latin America. Oxford: Oxford University Press.

Haggard, Stephan y Robert R. Kaufman. 1997. "The Political Economy of Democratic Transitions". The Journal of Comparative Politics 29 (3): 263-283. http://www.jstor.org/stable/422121

Haggard, Stephan. y Robert R. Kaufman. 1992. The Politics of Economic Adjustment. Princeton: Princeton University Press.

Revista Brasileira de Políticas Públicas e Internacionais, v.4, n.1, julho/2019, pp. 58-81. 
CIMINI, Fernanda; AGUIAR, Izabella Leal Miranda. El Giro a la Izquierda en el Uruguay de los Años 2000.

Huber, Evelyne y John D. Stephens. 2012. Democracy and the Left: Social Policy and Inequality. Chicago: University of Chicago Press.

Kaufman, Robert R. 2007. "The political effects of Inequality in Latin America: Some Inconvenient Facts". Ponencia presentada en el Second Annual International Conference Frontiers of Political Economics. State University, Russia.

Lanzaro, Jorge. 2011. "Uruguay. A Social Democratic Government in Latin America". En The resurgence of the Latin American left. Editado por Steven Levitsky y Kenneth Roberts, 348374. Baltimore: Johns Hopkins University Press.

Levitsky, Steven y Kenneth Roberts. 2011. The Resurgence of the Latin American Left. Baltimore: Johns Hopkins University Press.

Luna, Juan Pablo. 2002. “¿Pesimismo Estructural o Voto Económico?: Macropolitics en Uruguay". Revista Uruguaya de Ciencia Política 13: 123-152. https://www.colibri.udelar.edu.uy/jspui/handle/123456789/7139

Luna, Juan Pablo. 2007. "Frente Amplio and the Crafting of a Social Democratic Alternative in Uruguay". Latin American Politics and Society, 49 (4): 1-30. https://doi.org/10.1111/j.15482456.2007.tb00390.x

Luna, Juan Pablo. 2014. Segmented Representation. Political Party Strategies in Unequal Democracies. Oxford: Oxford University Press.

Mainwaring, Scott y Mariano Torcal. 2006. "Party System Institutionalization and Party System Theory after the Third Wave of Democratization". En Handbook of Party Politics. Editado por Richard Katz y William Crotty, 204-227. Thousand Oaks: Sage.

Melo, Caros Ranulfo. 2006. "Reforma política em perspectiva comparada na América do Sul". En Reforma política no Brasil. Editado por Leonardo Avritzer y Fátima Anastasia, 45-62. Belo Horizonte: Universidade Federal de Minas Gerais.

Muinelo-Gallo, Leonel y Adrián Rodríguez Miranda. 2014. "Descentralización fiscal, calidad de gestión de gobierno y disparidades regionales en Uruguay”. Estudios de Economía 41 (2): 219-250.

http://www.econ.uchile.cl/uploads/publicacion/e5b01f2b6f30f0b8f7c97ee7df5cfd6d4ad537b9.p df

Murillo, Maria Victoria. 2011. "Economic constraints and presidential agency". En The resurgence of the Latin American left. Editado por Steven Levitsky y Kenneth Roberts, 135174. Baltimore: Johns Hopkins University Press.

Revista Brasileira de Políticas Públicas e Internacionais, v.4, n.1, julho/2019, pp. 58-81. 
CIMINI, Fernanda; AGUIAR, Izabella Leal Miranda. El Giro a la Izquierda en el Uruguay de los Años 2000.

Pribble, Jennifer y Evelyne Huber. 2011. "Social Policy and Redistribution: Chile and Uruguay". En The resurgence of the Latin American left. Editado por Steven Levitsky y Kenneth Roberts, 117-138. Baltimore: Johns Hopkins University Press.

Przeworski, Adam. 2007. Political Rights, Property Rights, and Economic Development. Nova York: New York University.

Przeworski, Adam y Michael Wallerstein. 1982. "The structure of class conflict in democratic capitalist societies". American Political Science Review 76 (2): 215-238. http://www.jstor.org/stable/1961105

Queirolo, Rosario. 2006. "Las elecciones uruguayas de 2004: la izquierda como la única oposición creíble". Red Colombia Internacional (64): 34-49. http://www.scielo.org.co/scielo.php?script=sci_arttext\&pid=S012156122006000200003\&lng= en\&nrm=iso

Riella, Alberto y Alexandra Andrioli. 2004. "El Poder Simbólico de las Gremiales Ganaderos en el Uruguay Contemporáneo". Sociologias 6 (11): 184-218. http://www.scielo.br/pdf/soc/n11/n11a09.pdf

Rilla, José. 2008. "Uruguay 1985-2007: Restauración, Reforma, Crisis y Cambio Electoral”. Revista nuestra América (6): 63-95. https://bdigital.ufp.pt/bitstream/10284/2596/3/63-95.pdf

Santos, Manoel. 2011. "O parlamento sob influência: o lobby da indústria na Câmara dos Deputados”, tesis de doctorado, Universidade Federal de Pernambuco, Brasil.

Serna, Miguel. 2013. "Globalización, cambios en la estructura de poder y nuevas elites empresariales: una mirada comparada de Uruguay”. Revista de Sociologia e Política 21 (46): 93-103. http://dx.doi.org/10.1590/S0104-44782013000200006.

Silva, Fábio y Eleonora Cunha. 2014. "Process - Tracing e a Produção de Inferência Causal". En Teoria e Sociedade. Editado por Natália Sátyro y Maria Rodet, 104-125. Belo Horizonte: Universidade Federal de Minas Gerais.

Soifer, Hillel. 2012. "The Causal Logic of Critical Junctures". Comparative Political Studies 45(12): 1572-1597. http://journals.sagepub.com/doi/abs/10.1177/0010414012463902

Valenzuela, Arturo. 1977. Political Brokers in Chile: Local Government in a Centralized Polity. Durham: Duke University Press.

Weyland, Kurt, Raúl L. Madrid y Wendy Hunter. 2016. Leftist Governments in Latin America: successes and shortcomings. Cambridge: Cambridge University Press.

Revista Brasileira de Políticas Públicas e Internacionais, v.4, n.1, julho/2019, pp. 58-81. 